\title{
What happens to intimate partner violence studies registered on clinicaltrials.gov? A systematic review of a clinical trials registry
}

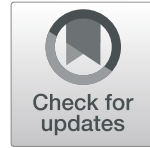

Kim Madden ${ }^{1,2^{*}}$ D, Kerry Tai ${ }^{1}$, Patricia Schneider ${ }^{1,3}$, Nathan Evaniew ${ }^{1}$, Michelle A. Ghert ${ }^{1}$ and Mohit Bhandari ${ }^{1,3}$

\begin{abstract}
Background: There is an increasing number of interventions aimed at reducing the incidence and improving the identification and management of intimate partner violence (IPV), which are being tested in randomized clinical trials. Publication bias, improper reporting, and selective reporting in clinical trials have led to widespread adoption of pre-registration of clinical trials. Non-publication of study results leads to inefficiency, ethical issues, and scientific issues with the IPV literature. When study results and methodology are not made available through publication or other public means, the results cannot be used to their full potential. The objective of this study was to determine the publication rates of IPV trials registered in a large clinical trial registry.

Methods: We conducted a systematic review of all IPV-related clinicaltrials.gov records and determined whether the studies that had been completed for $\geq 18$ months have been published in a peer-reviewed journal or in the clinicaltrials.gov registry. Two authors extensively searched the literature and contacted study investigators to locate full-text publications for each included study.
\end{abstract}

Results: Of 83 completed IPV-related trials registered on clinicaltrials.gov, 64 (77.1\%, 95\% Cl: 66.6-85.6) were subsequently published in full-text form. Of the 19 unpublished studies, authors confirmed that there was no publication for 11 studies; we were unable to contact the investigator or locate a publication for the remaining eight studies. Only four studies (all published) posted their results on clinicaltrials.gov upon completion.

Conclusion: Approximately one in four IPV trials are not published 18 months after completion, indicating that clinicians, researchers, and other evidence users should consider whether publication bias might affect their interpretation of the IPV literature. Further research is warranted to understand reasons for non-publication of IPV research and methods to improve publication rates.

Keywords: Intimate partner violence, Systematic review, Trial registration, Methodology, Publication bias

\section{Background}

Intimate partner violence (IPV), also known as spouse abuse and domestic violence, affects one in three women globally [1]. IPV is an important social issue that has well-documented health implications, including poor mental health [2] musculoskeletal injuries [3, 4] reduced quality of life [5], and even death in severe cases [6]. There is a growing number of interventions in healthcare settings for victims of IPV; these interventions are

\footnotetext{
* Correspondence: maddenk@mcmaster.ca

${ }^{1}$ Division of Orthopaedic Surgery, Department of Surgery, McMaster University, Hamilton, ON, Canada

${ }^{2}$ Research Institute of St. Joe's Hamilton, Hamilton, ON, Canada Full list of author information is available at the end of the article
}

increasingly being evaluated by clinical trials $[7,8]$. As the literature on IPV interventions grows, it is important to ensure transparency of study design and accurate trial reporting, and to evaluate potential bias in the literature, so that evidence users are not misled by inaccurate or inappropriate reporting. Additionally, since the effectiveness of IPV interventions is often highly controversial [9-11], it is important to have as much high-quality published evidence as possible.

It is important to register clinical trials for many reasons, including ethical obligations, legal obligations, and scientific considerations. Registering clinical trials allows patients and research participants to access information

(c) The Author(s). 2019 Open Access This article is distributed under the terms of the Creative Commons Attribution 4.0 International License (http://creativecommons.org/licenses/by/4.0/), which permits unrestricted use, distribution, and 
about clinical trials in which they could potentially participate (the registry's original purpose) [12]. Granting agencies and investigators can search trial registries to determine if there are any ongoing studies that might make a planned study redundant [12]. This usage aims to improve efficiency of clinical research and allocation of funding. Trial registries are also important for study methodology. Prospectively registering a study aims to reduce publication bias, selective reporting bias, and improve transparency [12]. Trial registries are publicly available databases, making it easy to find all trials that have been initiated for a particular intervention of interest. It is this transparency that should encourage investigators to publish their results regardless of whether they are positive, negative, or inconclusive, which has the potential to limit publication bias [13]. Because trial registry is required to occur before enrollment of the first patient, one can see in the trial record the originally planned eligibility criteria, intervention, comparison group, outcomes, and other important elements of the protocol. This means that registry records can be used to determine if the study plan changed over time so that the reader can assess if there is a risk of bias from selective reporting. Investigators are not currently required to register other study designs like observational studies, but it is encouraged.

Previous studies have reported very low rates of publication among studies registered on clincialtrials.gov and other trial registries. Ohnmeiss [14] found that only $38.9 \%$ of registered spine trials were published. Similarly, $22.8 \%$ of arthroplasty trials [15], $43.2 \%$ of trauma trials [16], 54\% of macular degeneration trials [17], 54\% of diagnostic accuracy studies [18], and $58.8 \%$ of sports medicine trials [19] are published. No previous studies have reported on the publication rates of registered studies in the IPV field. The current study can shed light on the current state of the IPV literature in terms of publication rates and potential for publication bias.

We conducted a systematic review of IPV studies registered with clinicaltrials.gov with the objective of determining the proportion of studies that have been published within 18 months of the trial being reported as complete on clinicaltrials.gov. Additionally, we aimed to explore the characteristics of trials that are published versus those that are not published.

\section{Methods}

\section{Identification of registry records}

We performed a search of the clinicaltrials.gov trial registry on 12 September 2017 using the terms "spouse abuse" OR "domestic violence" OR "partner violence" OR "partner abuse". Two authors (KM and KT) independently reviewed all study titles, outcomes, interventions, and conditions that the search identified. Studies were excluded if they focused only on child abuse, or if the title, outcomes, interventions, and conditions did not mention intimate partner violence or a related term such as domestic violence. We included all study designs (e.g. randomized trials, non-randomized studies, prospective cohort studies).

Once the relevant studies were identified, we determined whether the studies were "completed" or "not yet complete" based on what was reported in the clinicaltrials.gov record. At this point we excluded studies that were listed as "terminated," "withdrawn," or "suspended" in the registry. Additionally, we excluded studies with a date of completion in the past 18 months, in order to account for a reasonable time delay between trial completion and publication. We chose 18 months as our cutoff to allow sufficient time after the end of enrollment for data cleaning, data analysis, and manuscript writing, plus several months for review by a journal and subsequent publication. The World Health Organization (WHO) recommends publication within 12 months of study completion, but up to 24 months may be allowable [20]. Previous studies of publication rates of registered studies have used a cut-off of 18 months [14].

\section{Identification of publications}

We searched for each publication in AMED (Allied and Complementary Medicine Database), Embase, Global Health, Healthstar, Medline, and PsycInfo using the Ovid search interface, plus Google Scholar. We searched the clinicaltrials.gov trial identification number first; then, if the publication could not be found, we searched the publication databases using the principal investigator's (PI) last name plus trial keywords. An additional author (KT and PS) attempted to find the publications that the first author (KM) could not locate. We also attempted, on up to three occasions, to contact the PI listed on the clinicaltrials.gov record for publications that could not be located and for publications where we were unsure if they matched the clinicaltrials.gov record. We defined "publication" as a paper published in a peer-reviewed journal (i.e. not an internal report to industry, funding agency, or government). In addition, the publication had to contain results to be considered complete (protocol papers and initial reports were excluded).

\section{Data collection}

We exported the results of the clinicaltrials.gov search into a study database. For each study with a corresponding publication, one author (KM) extracted the month and year of publication, country, study design, intervention(s), funding source, and whether the authors reported the trial registry number. A second author 
(KT) verified all data points. Disagreements were settled by consensus or by consulting the senior author (MB).

\section{Data analysis}

We calculated agreement for inclusion using the kappa statistic with 95\% confidence interval (CI) using the GraphPad kappa calculator (http:/graphpad. com/quickcalcs/kappa2/). We used SPSS version 24 to conduct Fisher's exact tests and t-tests comparing unpublished and published study characteristics, and to construct a Kaplan-Meier survival curve for publication status (with an "event" defined as publication) and reported the median survival time with 95\% CI. We present descriptive statistics using frequencies and percentages, as appropriate. We also conducted a sensitivity analysis using a cut-off of 24 months since completion, per the upper limit of the WHO's recommendations for making study results available. We conducted an exploratory multivariable binary logistic regression to determine if country, study design, and funding source were associated with publication.

\section{Results}

Search results

We identified 274 study records in clinicaltrials.gov (Fig. 1). We excluded 59 of these studies because they did not relate to IPV and 106 because they were not yet completed. Four studies were withdrawn, suspended, or terminated; 22 had been completed $<18$ months before the registry search. Thus, there were 83 relevant clinicaltrials.gov records for which we sought matching publications. Inter-observer agreement for inclusion was almost perfect (kappa $=0.97,95 \%$ CI: 0.93-1.00).

\section{Published registered studies}

Of the 83 studies for which we sought full-text publications, we were able to locate 64 (77.1\%, 95\% CI: $66.6-$ 85.6). Of the remaining 19, authors of 11 studies confirmed that there is no publication; we were unable to contact the PI or to locate the publication for eight studies. Reasons given by authors for not having a published paper included that the publication is still in preparation or review, the results were uninteresting (i.e. negative), the study had methodological flaws, and the study was part of a $\mathrm{PhD}$ dissertation and was never published. Median time to publication was 32.0 months (95\% CI: 21.8-42.2) (Fig. 2) . Using a cut-off of 24 months since study completion, 60/ 77 studies were published (77.9\%).

\section{Study characteristics}

Study characteristics for published and unpublished studies are shown in Table 1 . Most studies were from the United States $(52 / 83,62.7 \%)$ and were randomized controlled trials (RCTs) $(66 / 83,79.5 \%)$.

Few studies $(4 / 83,4.8 \%)$ posted their results to clinicaltrials.gov. Interestingly, only $38 / 64$ published studies $(59.4 \%)$ reported their clinicaltrials.gov registration number in the published paper, despite that reporting the registration number is required by CONSORT guidelines [21]. We did not find any evidence that study design (RCT vs non-RCT; OR: 1.67, 95\% CI: 0.48-5.86) or country (USA vs non-USA; OR: $2.23,95 \%$ CI: 0.77-6.50) or funding source (Government/Non-Profit/Industry vs Unreported; OR: 2.817 , 95\% CI: 0.92-8.64) were associated with publication; however, with a small sample size, these results should be interpreted with caution.

\section{Discussion}

Clinicaltrials.gov and other trial registries are important tools to aid in transparency of conducting and reporting clinical research and reducing bias associated with nonpublication. Since IPV interventions and associated trials are a growing area of interest for clinicians and knowledge users, it is important to critically evaluate the quality of this body of literature in order to make informed decisions. This systematic review of clinicaltrials.gov records found that nearly one in four IPV-related studies are not published at 18 months or longer after being reported as completed on clinicaltrials.gov. The non-publication rate was nearly the same $(22.1 \%)$ when using a cut-off of 24 months instead of 18 months. There was no evidence that study design, country, or funding source are predictive of publication, but this finding should be interpreted with caution due to small numbers.

Publication bias is a well-documented phenomenon that arises when negative studies are not published and only positive studies are available to users of medical literature and systematic reviewers [22]. The effect is that interventions appear to be more effective than they actually are, thereby misleading clinicians and others seeking to apply results to clinical practice [22]. Some of the investigators contacted for the current review stated that they did not publish their study because they perceived that the study was not impactful (i.e. negative results), indicating the presence of publication bias. The most common reason for non-publication given by authors was that the paper was still in review at a journal. Although negative trials have similar [23] or better [24] methodological quality compared to positive trials, it often takes significantly longer for negative trials to be published compared to positive trials [25]. However, there is evidence that much of the decision not to publish negative trials is made by the author as opposed to journal editors in top medical journals [26]; therefore, authors must be aware of the consequences of publication bias 


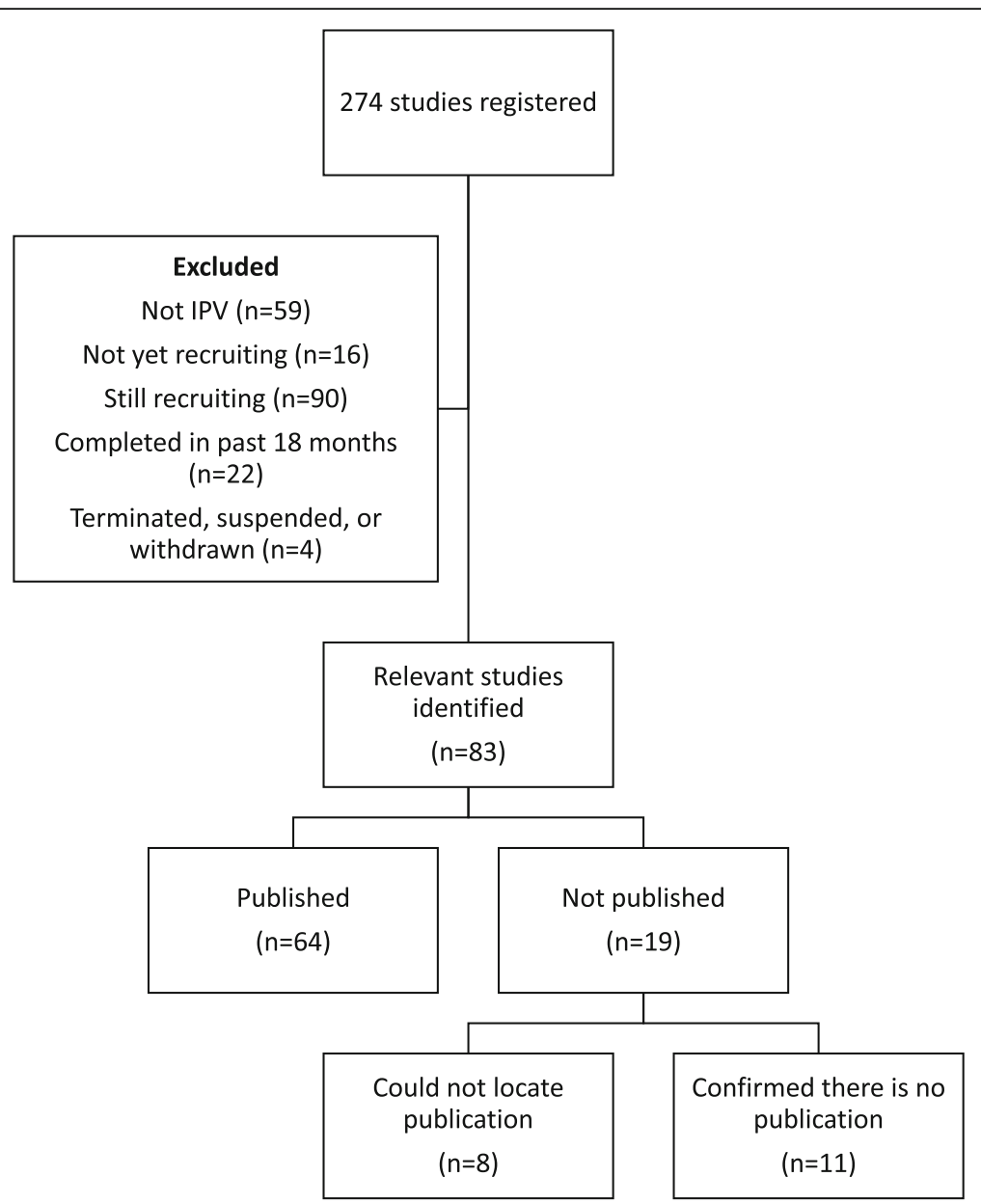

Fig. 1 Study flow diagram

and make all reasonable efforts to publish studies regardless of perceived impact or statistical significance.

Although there are other methods of making results of trials available, publication of study results in a peer-reviewed journal is the classic method of disseminating results to those who can use the knowledge in practice and in future research. Many other methods of dissemination are not publicly available except to a very select group of people (e.g. conference presentations, internal policy documents). Additionally, the full peer-reviewed publication usually contains the most comprehensive description of the study, allowing for proper critical appraisal and inclusion in knowledge syntheses. Since effective knowledge translation and exchange should an important goal of health research, by failing to publish studies, research funding is not used to its fullest potential.

Previous studies have reported very low publication rates in other fields. Ross et al. [27] randomly sampled $10 \%$ of all trials in a trials registry and found a publication rate of $<50 \%$. Similarly, with conference presentations, only $49 \%$ of poster and podium presentations in orthopedic surgery were published five years after presentation at the American Academy of Orthopedic Surgeons and 64\% after presentation at the Orthopedic Trauma Association $[28,29]$. It is also possible that other factors affect publication rate. For example, Hakala et al. [30] found that "stalled drugs" (i.e. drugs that reached late stage testing but were discontinued) had a publication rate of only $37 \%$ compared with licensed drugs that had a publication rate of $75 \%$. It is unclear whether there is a real difference between IPV research and other fields with respect to publication rates or if comparisons with other similar reviews are limited by differing methodologies.

A strength of this review is our exhaustive attempts to locate published studies using multiple techniques and multiple attempts to contact study investigators. Previous similar studies (e.g. [14, 19]) rarely attempted to contact investigators. This study has a few limitations as well. It is possible that some of the eight studies for which we were unable to locate a publication were actually published. However, the systematic and thorough 


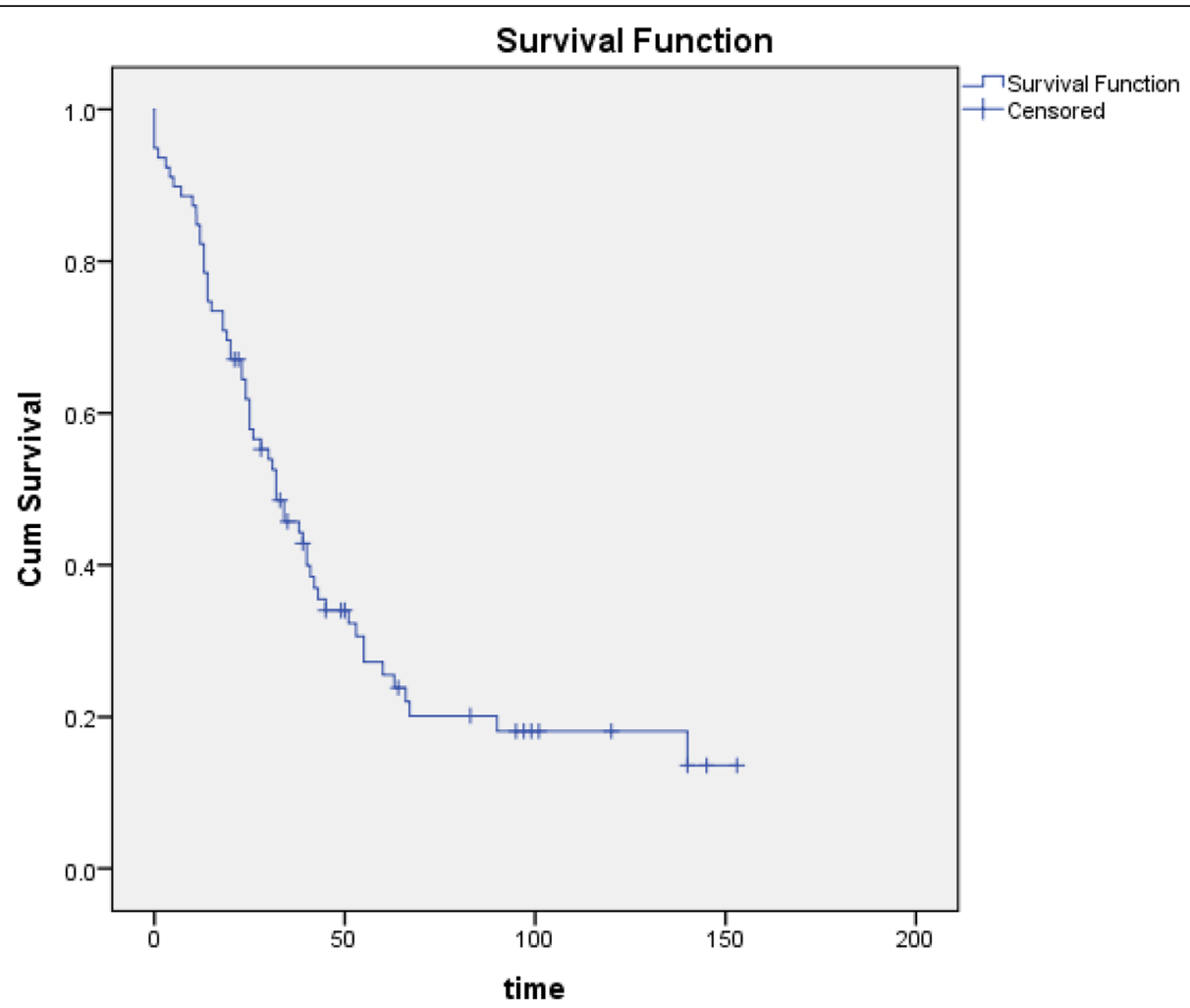

Fig. 2 Kaplan-Meier survival curve for time to publication

Table 1 Study characteristics

\begin{tabular}{lll}
\hline Study characteristic & $\begin{array}{l}\text { Published studies } \\
(N=64)\end{array}$ & $\begin{array}{l}\text { Unpublished studies } \\
(N=19)\end{array}$ \\
\hline $\begin{array}{l}\text { Study design } \\
\text { RCT }\end{array}$ & 52 & 14 \\
Non-RCT & 12 & 5 \\
Country & \\
USA & 43 & 9 \\
Other & 21 & 10 \\
Funding type & \\
Government & 43 & 11 \\
Foundation/Association & 7 & 0 \\
Industry & 1 & 0 \\
Unclear/Not reported & 13 & 8 \\
Results reported in registry & & \\
Yes & 4 & 19 \\
No & 60 & \\
NCT number reported in publication & 38 & \\
Yes & 26 & \\
No & & \\
\hline
\end{tabular}

design of this review with comprehensive searching, double-checking, and contact with investigators attempts to minimize this possibility. Current recommendations for systematic reviews suggest searching Medline, Embase, and the Cochrane Register at minimum [31]. We exceeded this minimum recommendation in our search strategy, enhancing the strength of our conclusions. It is also a possibility that some of the eight studies that we were unable to locate were published in gray literature or journals that are not indexed in major databases, but our conclusions would remain the same, since such publications would not be easily accessible by a general user of medical literature.

We were unable to determine the association between industry funding and non-publication due to small numbers. Future research could investigate the impact of industry funding on IPV studies. We were unable to determine whether statistical significance (i.e. a positive versus negative trial) was related to non-publication because it is not possible to determine the statistical significance of unpublished studies, so we cannot make comparisons between published and unpublished studies. We did not examine the quality of the literature because the primary outcome was non-publication. It is not possible to evaluate the quality of studies that are not published. Additionally, we were able to gather only limited data 
on reasons why studies are not published in IPVrelated research as it was outside the scope of this study; however, it warrants further research. There may be reasons unique to IPV research why studies are not published. For example, members of the current study team experienced rejection of a publication when we attempted to publish in a specialized surgery journal because the editor did not believe that IPV is a surgeon's issue.

\section{Conclusions}

Approximately one in four registered IPV studies are not published following completion, which means that clinicians, researchers, and other evidence users should consider whether publication bias might affect their interpretation of the IPV literature. Publication bias in IPV literature could lead to an over-estimation of the effectiveness of IPV interventions which could mislead clinicians and policymakers. Additionally, the non-publication of completed IPV studies indicates that research funding is wasted. Further research is warranted to understand reasons for non-publication of IPV research and methods to improve publication rates. Investigators of completed studies as well as journal editors should be aware of the consequences of publication bias.

\begin{abstract}
Abbreviations
AMED: Allied and Complementary Medicine Database; Cl: Confidence interval; CIHR: Canadian Institutes of Health Research; CONSORT : Consolidated Standards of Reporting Trials; FDA: Food and Drug Administration; FDAAA: Food and Drug Administration Amendments Act; GRADE: Grading of Recommendations Assessment, Development and Evaluation; ICMJE: International Committee of Medical Journal Editors; IPV: Intimate partner violence; LMIC: Low- and middleincome countries; NIH: National Institutes of Health; OR: Odds ratio; PI: Principal investigator; RCT: Randomized controlled trial; WHO: World Health Organization
\end{abstract}

\section{Acknowledgements}

Not applicable.

\section{Authors' contributions}

$\mathrm{KM}$ and $\mathrm{MB}$ designed the study. KM, KT, and PS collected data. All authors analyzed and/or interpreted data. KM drafted the manuscript. All authors made significant revisions and approved the final manuscript.

\section{Funding}

Ms. Madden is funded by a Canadian Institutes of Health Research (CIHR) Doctoral award. Dr. Bhandari is funded, in part, by a Canada Research Chair. No funding was received for the preparation of this manuscript. The funders had no role in the design of the study, collection, analysis, interpretation of data or in writing the manuscript.

\section{Availability of data and materials}

The datasets used and/or analyzed during the current study are available from the corresponding author on reasonable request.

\section{Ethics approval and consent to participate}

Not applicable.

\section{Consent for publication}

Not applicable.

\section{Competing interests}

The authors declare that they have no competing interests.

\section{Author details}

1Division of Orthopaedic Surgery, Department of Surgery, McMaster University, Hamilton, ON, Canada. ${ }^{2}$ Research Institute of St. Joe's Hamilton, Hamilton, ON, Canada. ${ }^{3}$ Department of Health Research Methods, Evidence, and Impact, McMaster University, Hamilton, ON, Canada.

Received: 18 October 2017 Accepted: 7 May 2019

Published online: 27 May 2019

\section{References}

1. World Health Organization. WHO multi-country study on women's health and domestic violence against women. Geneva: WHO; 2005. http://www.who.int/gender/violence/who multicountry study/Chapter7Chapter8-Chapter9.pdf

2. Ellsberg M, Jansen HA, Heise L, Watts CH, Garcia-Moreno C, WHO Multicountry Study on Women's Health and Domestic Violence against Women Study Team. Intimate partner violence and women's physical and mental health in the WHO multi-country study on women's health and domestic violence: an observational study. Lancet. 2008;371:1165-72.

3. Bhandari M, Dosanjh S, Tornetta P 3rd, Matthews D, Violence Against Women Health Research Collaborative. Musculoskeletal manifestations of physical abuse after intimate partner violence. J Trauma. 2006;61 (6):1473-9.

4. Wu V, Huff H, Bhandari M. Pattern of physical injury associated with intimate partner violence in women presenting to the emergency department: a systematic review and meta-analysis. Trauma Violence Abuse. 2010;11(2):71-82.

5. Dillon G, Hussain R, Loxton D, Rahman S. Mental and physical health and intimate partner violence against women: a review of the literature. Int J Family Med. 2013;2013:313909. https://doi.org/10.1155/2013/313909.

6. Domestic Violence Death Review Committee 2017 Annual Report. http:// cdhpi.ca/sites/cdhpi.ca/files/2017-DVDRC-Report.pdf.

7. Coker AL, Smith PH, Whitaker DJ, Le B, Crawford TN, Flerx VC. Effect of an in-clinic IPV advocate intervention to increase help seeking, reduce violence, and improve well-being. Violence Against Women. 2012;18:118.

8. Klevens J, Kee R, Trick W, Garcia D, Angulo FR, Jones R, et al. Effect of screening for partner violence on women's quality of life: A randomized controlled trial. JAMA. 2012;308:681.

9. U.S. Preventive Services Task Force. Screening for family and intimate partner violence: recommendation statement. Ann Fam Med. 2004; 2(2):156-60.

10. MacMillan $\mathrm{HL}$, Feder G. Screening women for intimate partner violence. Ann Intern Med. 2012:157(9):676 author reply 676-7.

11. Moyer VA, U.S. Preventive Services Task Force. Screening for intimate partner violence and abuse of elderly and vulnerable adults: U.S. Preventive Services Task Force recommendation statement. Ann Intern Med. 2013;158(6):478-86

12. Zarin DA, Keselman A. Registering a clinical trial in ClinicalTrials.gov. Chest. 2007;131(3):909-12.

13. Abaid LN, Grimes DA, Schulz KF. Reducing publication bias through trial registration. Obstet Gynecol. 2007:109(6):1434-7.

14. Ohnmeiss DD. The fate of prospective spine studies registered on www. ClinicalTrials.gov. Spine J. 2015:15:487-91.

15. Smith HN, Bhandari M, Mahomed NN, Jan M, Gandhi R. Comparison of arthroplasty trial publications after registration in Clinical-Trials.gov. J Arthroplast. 2012;27:1283-8.

16. Gandhi R, Jan M, Smith HN, Mahomed NN, Bhandari M. Comparison of published orthopaedic trauma trials following registration in Clinicaltrials. gov. BMC Musculoskelet Disord. 2011;12:278.

17. Prenner JL, Driscoll SJ, Fine HF, Salz DA, Roth DB. Publication rates of registered clinical trials in macular degeneration. Retina. 2011;31(2):401-4.

18. Korevaar DA, Ochodo EA, Bossuyt PM, Hooft L. Publication and reporting of test accuracy studies registered in ClinicalTrials.gov. Clin Chem. 2014;60(4):651-9.

19. Chahal J, Tomescu SS, Ravi B, Bach BR, Ogilvie-Harris D, Mohamed NN, et al. Publication of sports medicine-related randomized controlled trials registered in ClinicalTrials.gov. Am J Sports Med. 2012;40:1970-7.

20. World Health Organization. International Standards For Clinical Trial Registries. Geneva: WHO; 2012. http://apps.who.int/iris/bitstream/10665/76705/1/97892 41504294_eng.pdf?ua $=1 \& u a=1$

21. Schulz KF, Altman DG, Moher D, for the CONSORT Group. CONSORT 2010 Statement: updated guidelines for reporting parallel group randomised trials. BMJ. 2010;340:C332.

22. Dickersin K, Chan S, Chalmers TC, Sacks HS, Smith H Jr. Publication bias and clinical trials. Control Clin Trials. 1987;8(4):343-53. 
23. Charan J, Chaudhari M, Jackson R, Mhaskar R, Reljic T, Kumar A. Comparison of methodological quality of positive versus negative comparative studies published in Indian medical journals: a systematic review. BMJ Open. 2015;5(6):e007853.

24. Chiavetta NM, Martins AR, Henriques IC, Fregni F. Differences in methodological quality between positive and negative published clinical trials. J Adv Nurs. 2014;70(10):2389-403.

25. Ioannidis JP. Effect of the statistical significance of results on the time to completion and publication of randomized efficacy trials. JAMA. 1998:279(4):281-6.

26. van Lent M, Overbeke J, Out HJ. Role of editorial and peer review processes in publication bias: analysis of drug trials submitted to eight medical journals. PLoS One. 2014;9(8):e104846.

27. Ross JS, Tse T, Zarin DA, Xu H, Zhou L, Krumholz HM. Publication of NIH funded trials registered in ClinicalTrials.gov: cross sectional analysis. BMJ. 2012;344:d7292.

28. Donegan DJ, Kim TW, Lee GC. Publication rates of presentations at an annual meeting of the american academy of orthopaedic surgeons. Clin Orthop Relat Res. 2010;468(5):1428-35.

29. Nguyen V, Tornetta P 3rd, Bkaric M. Publication rates for the scientific sessions of the OTA. Orthopaedic Trauma Association. J Orthop Trauma. 1998;12(7):457-9 discussion 456.

30. Hakala A, Kimmelman J, Carlisle B, Freeman G, Fergusson D. Accessibility of trial reports for drugs stalling in development: a systematic assessment of registered trials. BMJ. 2015;350:h1116.

31. Murad MH, Montori VM, loannidis JP, Jaeschke R, Devereaux PJ, Prasad $K$, et al. How to read a systematic review and meta-analysis and apply the results to patient care: users' guides to the medical literature. JAMA. 2014;312(2):171-9.

\section{Publisher's Note}

Springer Nature remains neutral with regard to jurisdictional claims in published maps and institutional affiliations.

Ready to submit your research? Choose BMC and benefit from:

- fast, convenient online submission

- thorough peer review by experienced researchers in your field

- rapid publication on acceptance

- support for research data, including large and complex data types

- gold Open Access which fosters wider collaboration and increased citations

- maximum visibility for your research: over $100 \mathrm{M}$ website views per year

At $\mathrm{BMC}$, research is always in progress.

Learn more biomedcentral.com/submissions 